\title{
ON SOME INTEGRAL REPRESENTATIONAL FORMULAS FOR SCHWARZIAN COEFFICIENTS WITH AN APPLICATION TO NUMBER THEORY
}

\author{
STEPHEN M. ZEMYAN
}

\begin{abstract}
A.bstract. We develop integral representational formulas for all Schwarzian coefficients of single-slit mappings by utilizing Löwner's Parametric Method. As an application, we evaluate a complicated numbertheoretic sum.
\end{abstract}

Let $S$ denote the class of functions $f(z)=z+a_{2} z^{2}+\cdots$ which are analytic and univalent in the unit disk $U=\{z:|z|<1\}$.

The Schwarzian derivative of a function $f(z)$ in $S$ is defined by the relation

$$
\{f, z\}=\left(\frac{f^{\prime \prime}(z)}{f^{\prime}(z)}\right)^{\prime}-\frac{1}{2}\left(\frac{f^{\prime \prime}(z)}{f^{\prime}(z)}\right)^{2}
$$

and the Schwarzian coefficients of the function $f(z)$ are the Taylor coefficients in the series expansion

$$
\{f, z\}=\sum_{n=0}^{\infty} s_{n} z^{n}
$$

The goal of this paper is to develop integral representational formulas for all Schwarzian coefficients of single-slit mappings by utilizing Löwner's parametric 
method, and to give a number-theoretic application of these formulas. We follow the development given in $[8, p .156-164]$, aithough the notation varies slightly.

A function $g(z, t)=e^{t} z+a_{2} z^{2}+\cdots$ is called a Löwner Chain if it is analytic and univalent in $U$ for each $t \in[0, \infty)$, and if $g(U, s) \subseteq g(U, t)$ whenever $0 \leq s \leq t<+\infty$. Since the functions $f(z, t)=e^{-t} g(z, t)$ belong to $S$ for each $t \in[0, \infty)$, we shall refer to $f(z, t)$ as a normalized Löwner Chain in $S$.

For every $f \in S$, there exists a Löwner Chain $g(z, t)$ such that $f(z)=$ $g(z, 0)$. The main result of the Löwner Theory states that the functions $g(z, t)$ are absolutely continuous in $t$. Furthermore, it also states that there exists a function $p(z, t)=1+p_{1}(t) z+\cdots$ which is analytic in $U$ and measurable in the variable $t$ satisfying

$$
\operatorname{Re} p(z, t)>0 \quad(z \in U, t \in[0, \infty))
$$

such that, for almost all $t \in[0, \infty)$,

$$
\frac{\partial}{\partial t} g(z, t)=z p(z, t) \frac{\partial}{\partial z} g(z, t)
$$

We use the Löwner method to prove

Theorem 1. Let $f(z, t)$ be a normalized Löwner Chain in S. Define

$$
\Phi(z, \zeta, t)=\log \left(\frac{f(z, t)-f(\zeta, t)}{z-\zeta}\right) \quad(z, \zeta \in U, t \in[0, \infty))
$$

Then $\Phi$ satisfies the partial differential equation*

$$
1+\frac{\partial \Phi}{\partial t}=z p(z, t) \frac{\partial \Phi}{\partial z}+\zeta p(\zeta, t) \frac{\partial \Phi}{\partial \zeta}+\frac{z p(z, t)-\zeta p(\zeta, t)}{z-\zeta}
$$

for almost every $t$, where $p(\cdot, t)$ is the function of positive real part which appears in the Löwner equation (3).

* The author is currently preparing a monograph exploring the numerous consequences of this equation for the Grunsky coefficients of a univalent function. 
Proof. Replace $z$ with $\zeta$ in (3) to obtain

$$
\frac{\partial}{\partial t} g(\zeta, t)=\zeta p(\zeta, t) \frac{\partial}{\partial \zeta} g(\zeta, t)
$$

Now, subtract (6) from (3) and multiply by $e^{-t}$ to obtain

$$
\begin{aligned}
& f(z, t)-f(\zeta, t)+\frac{\partial}{\partial t}(f(z, t)-f(\zeta, t)) \\
= & z p(z, t) \frac{\partial}{\partial z}(f(z, t)-f(\zeta, t))+\zeta p(\zeta, t) \frac{\partial}{\partial \zeta}(f(z, t)-f(\zeta, t)) .
\end{aligned}
$$

To complete the proof, divide by $f(z, t)-f(\zeta, t)$ and insert $\log (z-\zeta)$ where necessary.

From (5), it is easy to obtain a Löwner-type equation for the Schwarzian derivative of a normalized Löwner Chain in $S$.

Theorem 2. Let $f(z, t)$ be a normalized Löwner chain in $S$. Then, the function

$$
\Gamma(z, t)=z^{2}\{f(z, t), z\}=\sum_{n=0}^{\infty} s_{n}(t) z^{n+2}
$$

satisfies

$$
\frac{\partial \Gamma}{\partial t}=z p(z, t) \frac{\partial \Gamma}{\partial z}+2 z p^{\prime}(z, t) \Gamma(z, t)+z^{2} \frac{\partial^{3}}{\partial z^{3}}(z p(z, t))
$$

for almost every $t$, where $p(z, t)$ is determined from the Löwner equation (3).

Proof. Differentiating (5) with respect to $z$ and $\zeta$, we obtain

$$
\begin{aligned}
\Phi_{z \zeta t}= & p(z, t)\left(\Phi_{z \zeta}+z \Phi_{z \zeta z}\right)+p(\zeta, t)\left(\Phi_{z \zeta}+\zeta \Phi_{z \zeta \zeta}\right) \\
& +z p^{\prime}(z, t) \Phi_{z \zeta}+\zeta p^{\prime}(\zeta, t) \Phi_{z \zeta}+\left(\frac{z p(z, t)-\zeta p(\zeta, t)}{z-\zeta}\right)_{z \zeta} .
\end{aligned}
$$

Recalling the fact that

$$
\{f, z\}=6 \cdot \lim _{\zeta \rightarrow z} \frac{\partial^{2} \Phi(z, \zeta)}{\partial z \partial \zeta}
$$


we let $\zeta \rightarrow z$ to complete the proof.

Corollary 2.1. Each Schwarzian coefficient $s_{n}(t)$ of the normalized Löwner chain $f(z, t)$ satisfies a first order recursive ordinary differential equation. Specifically, for each $n \geq 0$,

$$
s_{n}^{\prime}(t)=(n+1)(n+2)(n+3) p_{n+2}(t)+\sum_{k=0}^{n}(2 n+2-k) s_{k}(t) P_{n-k}(t)
$$

for almost every $t$, where $p_{m}(t)$ are coefficients in the series expansion of $p(z, t)$.

Proof. Substitute the series representations for $\Gamma$ and $p$ into (8) and equate coefficients.

Remark. A system of ordinary differential equations similar to (9) was directly used by Fitzgerald and Pommerenke [13] in an alternate proof of de Branges Theorem. In view of their work, it is natural to ask whether the system (9) may be used successfully to establish inequalities of the form

$$
\sum_{n=0}^{N} \alpha_{n}\left|s_{n}\right|^{2} \leq \sum_{n=0}^{N} \beta_{n} \sigma_{n}^{2}
$$

where $\alpha_{n}, \beta_{n}$ and $\sigma_{n}$ are fixed positive constants. The author has met with limited success in attempting to do so.

If $N=0$, their method quickly yields $\left|s_{0}\right|^{2} \leq 36$. If $N=1$, their method may again be employed to show that $\left|s_{0}\right|^{2}+\alpha\left|s_{1}\right|^{2} \leq 36$ for all $\alpha \in[0,3 / 32]$, which is not the best possible inequality. If $N \geq 2$, the algebraic forms of the system prevents further progress.

We are now ready to show that the system of Corollary 2.1 may be resolved to obtain integral representations for all Schwarzian coefficients $s_{n}(t)$ of a normalized Löwner chain.

Theorem 3. Let $f(z, t)$ be a normalized Löwner chain in $S$, and let the Schwarzian coefficients of $f(z, t)$ be defined by the relation $\{f(z, t), z\}=$ 
$\sum_{n=0}^{\infty} s_{n}(t) z^{n}$. Then, for all $n=0,1,2, \ldots$, each $s_{n}(t)$ admits the representation.

$$
s_{n}(t)=e^{(n+2) t} \sum_{m=1}^{n+1}(-1)^{m} U_{n}(m ; t)
$$

where the inner sum

$$
U_{n}(m ; t)=\frac{1}{2} \sum_{\pi_{n+2}(m)}\left(n_{1}^{2}-1\right) G\left(n_{m}, \ldots, n_{1}\right) F\left(p ; n_{m}, \ldots, n_{1} ; t\right)
$$

is taken over the set $\pi_{n+2}(m)$ of all possible sums of the form $n+2=n_{1}+\cdots+n_{m}$ composed of exactly $m$ positive integers, with $n_{1} \geq 2$. Here, for positive integers $n_{1}, \cdots, n_{m}$, we have defined

$$
G\left(n_{m}, \cdots, n_{1}\right)=\prod_{r=1}^{m}\left(n_{r}+\sum_{i=1}^{r} n_{i}\right)
$$

and

$$
\begin{aligned}
& F\left(p ; n_{m}, \cdots, n_{1} ; t\right) \\
= & \int_{t}^{\infty} \int_{t m}^{\infty} \cdots \int_{t_{2}}^{\infty}\left[\prod_{i=1}^{m} p_{n_{i}}\left(t_{i}\right) e^{-n_{i} t_{i}}\right] d t_{1} \ldots d t_{m-1} d t_{m_{n}}
\end{aligned}
$$

where $p_{n_{i}}(t)(i=1, \ldots, m)$ are coefficients in the series expansion of $p(z, \hat{i})$.

Proof. (Weak induction.) Let $n=0$. A simple integration shows that the differential equation

$$
s_{0}^{\prime}(t)=6 p_{2}(t)+2 s_{0}(t)
$$

has the solution

$$
s_{0}(t)=-6 e^{2 t} F(p ; 2 ; t)
$$

On the other hand,

$$
\begin{aligned}
s_{0}(t) & =-e^{2 t} U_{0}(1 ; \iota) \\
& =-\frac{1}{2} e^{2 t} \cdot\left(2^{2}-1\right) \cdot G(2) F(p ; 2 ; t) \\
& =-6 e^{2 t} F(p ; 2 ; t),
\end{aligned}
$$


so that our claim is valid if $n=0$.

Now, let $n \geq 1$, and assume that the claimed representation is valid for all non-negative integers up to and including $n-1$. Multiply (9) by $e^{-(n+2) t}$ and integrate the result from $t$ to $\infty$ to obtain

$$
\begin{aligned}
s_{n}(t)=-e^{(n+2) t}\left[(n+1)(n+2)(n+3) \int_{t}^{\infty} p_{n+2}(s) e^{-(n+2) s} d s\right. \\
\left.\quad+\sum_{j=0}^{n-1}(2 n+2-j) \int_{t}^{\infty} s_{j}(s) p_{n-j}(s) e^{-(n+2) s} d s\right] \\
=-e^{(n+2) t} \sum_{\pi_{n+2}(1)} \frac{1}{2}\left((n+2)^{2}-1\right) G(n+2) F(p ; n+2 ; t) \\
\left.\quad+\sum_{j=0}^{n-1}(2 n+2-j) \sum_{m=1}^{j+1}(-1)^{m} \int_{t}^{\infty} U_{j}(m ; s) p_{n-j}(s) e^{-(n-j) s} d s\right]
\end{aligned}
$$

For each $j$ and $m$, we set $p_{m+1}=N-j$. Then,

$$
\begin{aligned}
& \int_{t}^{\infty} U_{j}(m ; s) p_{n-j}(s) e^{-(n-j) s} d s \\
= & \sum_{\pi_{j+2}(m)} \frac{1}{2}\left(p_{1}^{2}-1\right) G\left(n_{m}, \ldots, n_{1}\right) \\
& \cdot \int_{t}^{\infty} F\left(p ; n_{m}, \ldots, n_{1} ; s\right) p_{n-j}(s) e^{-(n-j) s} d s \\
= & \sum_{\pi_{j+2}(m)} \frac{1}{2}\left(p_{1}^{2}-1\right) G\left(n_{m}, \ldots, n_{1}\right) F\left(p ; p_{m+1}, p_{m}, \ldots, p_{1} ; t\right)
\end{aligned}
$$

where $n_{1}+\ldots+n_{m}=j+2$ and $n_{1}+\ldots+n_{m+1}=n+2$. Observe that $G\left(n_{m+1}, \ldots, n_{1}\right)=(2 n+2-j) G\left(n_{m}, \ldots, n_{1}\right)$. If we now substitute (15) into (14), interchange the order of summation, and shift indices, then our representational formula becomes

$$
\begin{aligned}
s_{n}(t)= & e^{(n+2) t}\left[(-1) U_{n}(1 ; t)+\sum_{m=2}^{n+1}(-1)^{m}\right. \\
& \left.\cdot\left(\sum_{j=m-2}^{n-1} \sum_{\pi_{j+2}(m-1)} \frac{1}{2}\left(p_{1}^{2}-1\right) G\left(n_{m}, \ldots, n_{1}\right) F\left(p ; n_{m}, \ldots, n_{1} ; t\right)\right)\right] .
\end{aligned}
$$


It remains to see that this double sum is actually equal to $U_{n}(m ; t)$. This will be clear if we realize that set $\pi_{n+2}(m)$ of sums of the form $n_{1}+\ldots+n_{m}=n+2$ may be partitioned into equivalence classes according to the value of $n_{m}$. If $n_{1}+\ldots+n_{m-1}=j+2$ and $n_{m}=n-j$, then $n_{1}+\ldots+n_{m}=n+2$; and, as $j$ increases from $(m-2)$ to $(n-1), n_{m}$ decreases form $n+2-m$ to 1 . If $n_{m}>n+2-m$, then $n_{1}+\ldots+n_{m-1}<m$, with $n_{1} \geq 2$ and $n_{j} \geq 1$. Since this is impossible, all values of $n_{m}$ have been accounted for in this sum, and the proof is complete.

Remark. If $f$ maps $U$ onto the complex plane less a Jordan arc, then $p$ takes the form $[2$, p. 93]

$$
p(z, t)=\frac{1+k(t) z}{1-k(t) z}=1+\sum_{m=1}^{\infty} 2 k^{m}(t) z^{m}
$$

where $k(t)$ is a continuous, complex-valued function on $[0, \infty)$ with $|k(t)| \equiv 1$. Both the equations (5), (8) and (9), and the representational formulas (11) and (13) could easily be adjusted to reflect this choice for $f(z)$. Thus,

$$
F\left(k ; n_{m}, \ldots, n_{1} ; t\right)=2^{m} \int_{t}^{\infty} \int_{t_{m}}^{\infty} \ldots \int_{t_{2}}^{\infty}\left[\prod_{i=1}^{m} k^{n_{i}}\left(t_{i}\right) e^{-n_{i} t_{i}}\right] d t_{1} \ldots d t_{m-1} d t_{m} .
$$

If, in particular, $k(t) \equiv 1$ on $[0, \infty)$ and $t=0$, then

$$
F\left(1 ; n_{m}, \ldots, n_{1} ; 0\right)=\frac{2^{m}}{\prod_{r=1}^{m}\left(\sum_{i=1}^{r} n_{i}\right)} .
$$

We use this observation to provide a rather startling number-theoretic consequence of Theorem 3.

Corollary 3.1. For positive integers $n_{1}, \ldots, n_{m}$, let

and

$$
H\left(n_{1}, \ldots, n_{m}\right)=\prod_{r=1}^{m}\left(\sum_{i=1}^{r} n_{i}\right)
$$




$$
G\left(n_{1}, \ldots, n_{m}\right)=\prod_{r=1}^{m}\left(n_{r}+\sum_{i=1}^{r} n_{i}\right) .
$$

For integers $n \geq 0$ and $m \geq 1$, define

$$
U_{n}(m)=\sum_{\pi_{n+2}(m)} \frac{1}{2}\left(p_{1}^{2}-1\right) \frac{G\left(n_{1}, \ldots, n_{m}\right)}{H\left(n_{1}, \ldots, n_{m}\right)}
$$

where the sum is taken over all possible sums of the form $n_{1}+\ldots+n_{m}=n+2$, with $n_{1} \geq 2$. If we set

$$
S_{n}=\sum_{m=1}^{n+1}(-2)^{m} U_{n}(m)
$$

then, for all $k=0,1,2,3, \ldots$,

$$
S_{2 k}=-6(k+1) \quad \text { and } \quad S_{2 k+1}=0
$$

Proof. Since we expect very limited cancellation in the quotients $G / H$, it is very surprising that the values $S_{n}$ are integers!

To prove this fact, we choose $f(z)=z /(1+z)^{2}$. On one hand, its Schwarzian coefficients are given by (17). On the other hand, $f(z)=f(z, t)=e^{-t} g(z, t)$ for all $t \in[0, \infty)$. Form (3), we deduce that $p(z, t)=(1+z) /(1-z)$ and that $k(t) \equiv 1$ on $[0, \infty)$. Now, merely compute $s_{n}(0)=S_{n}$ using Theorem 3 and formula (16).

Remark. It is also possible to give representational formulas of this type for the coefficients of single-slit mappings in $\sum$, the logarithmic coefficients of $f$, the logarithmic coefficients of $f^{\prime},(f \in S)$, etc. along a normalized Löwner chain. These formulas will have number-theoretic corollaries as well.

\section{References}

[1] P.L. Duren, "Univalent Functions", Springer-Verlag, Heidelberg and New York, 1983.

[2] G.M. Goluzin, "Geometric Theory of Functions of a Complex Variable", English Transl., Amer. Math Soc., Providence, R.I., 1969.

[3] R. Harmelin, "Generalized Grunsky coefficients and inequalities", Israel J. of Math., Vol. 57, No. 3. (1987), 347-364.

[4] E. Hille, "Analytic Functionn Theory", II, Chelsea, New York, 1962. 
[5] J.A. Hummel, "The Grunsky coefficients of a schlicht function", Proc. Amer. Math. Soc., 15 (1964), 142-150.

[6] J.A. Jenkins, "On certain coefficients of univalent functions in Analytic Functions", Princeton Univ. Press, Princeton, N.J., 1960.

[7] W. Kraus, "Üger den Zusammenhang einiger Charakteristiken eines einfach zusammenhängenden Bereiches mit der Kreisabbildung", Mitt. Math. Sem. Giessen, 21 (1932), 1-28.

[8] Ch. Pommerenke, "Univalent Functions", Vandenhoeck and Ruprecht, Göttingen, 1975.

[9] M. Schiffer, "Sur un problème d'extremum de la représentation conforme", Bull. Soc. Math. France, 66 (1938), 48-55.

[10] G. Schober, "Univalent Functions-Selected Topics", Lecture Notes in Math. No. 478, Springer-Verlag, 1975.

[11] I. Schur, "On Faber Polynomials", Amer. J. Math., 67 (1945), 33-41.

[12] P. Todorov, "Explicit Formulas for the cofficients of Faber polynomials with respect to univalent functions of the class $\sum$ ", Proc. Amer. Math. Soc., Vol. 82, Number 3, (1981), 431-438.

[13] C. Fitzgerald and Ch. Pommerenke, "The de Branges theorem on univalent functions", Trans. Amer. Math. Soc., 290 (1985), 683-690.

[14] S. Zemyan, "On the Schwarzian Coefficients of Univalent Functions", Bull. Austral. Math. Soc., Vol. 46 (1992), 389-198.

Department of Mathematics, The Pennsylvania State University, Mont Alto Campus, Mont Alto, Pennsylvania 17237-9799, U.S.A. 